\title{
Double Approach in the Investigation of Light Scattering by a Nickel Metal Cylinder
}

\author{
A. Kazadi Mukenga Bantu, T. Mabiala Masiala, E. Phuku Phuati, \\ J.P. Mbungu Tsumbu, and P. Defrance
}

\begin{abstract}
In the present work, we have investigated the light scattering from a Nickel metal cylinder under normal incidence. A double approach using the Fresnel reflectance model and Lorenz-Mie approach shows that the Fresnel model cannot follow the changes in the scattering regime when the size parameter changes. Experiments show that, even for large cylinders, the Fresnel model overestimates the effects of reflection in the forward direction. The simulation using the Lorenz-Mie formalism shows that as the size parameter changes the scattering regime changes and for size parameter lower than one the distribution of light intensity tends to become uniform and, for size parameter equal or lower than 0.1 the distribution is uniform and independent on the scattering angle, with a strong polarization around $70 \%$.
\end{abstract}

Keywords - Backscattering, Light Scattering, Nano-optics.

\section{INTRODUCTION}

Understanding and controlling the properties of light scattering is of importance for various applications such as optical antennas, optical switching, sensing, photo-detection, light emission control, and solar cells [1]. Cylinders are common geometries that are found in the design of many devices [2] such as antennas, resonators, etc., moreover many natural structures can be modeled as cylinders (biological tissues, ice clouds...). The study of light scattering by such structures is of great importance in many aspects. Actually, with the advent of nanosciences it is important to study the behavior of such geometries when their dimensions reach or become smaller than the incident wavelength. On the other hand, it is also important to study the scattering regime when the dimensions are very large in comparison to the wavelength of the incident radiation. Among the multiple characteristics of the scattered field, there are its intensity distribution and polarization. We have systematically studied these two components for a nickel metallic cylinder.

The light scattering by cylindrical bodies is of great interest in many disciplines. In the present work, we focus on the light scattering by a Nickel cylinder in the air; under normal incidence. We investigated the scattering through the Lorenz-Mie model, and we compared it with the Fresnel model. A simulation code is written using MATLAB software for the above named model, another code using the Fresnel reflection coefficients is also written for Transverse Magnetic (TM) and Transverse Electric (TE) modes. From the intensity distribution for each of the polarization the linear polarization is deduced. Finely we compare the Fresnel model with the experiment, using a cylinder which diameter is very large in comparison to the incident wavelength.

The rest of the paper is organized as follows. Section II provides a brief introduction to the theory, simulation, and experiment of our body. In Section III, we present results and discussion on the scattering of the Transverse Magnetic (TM) and Transverse Electric (TE) modes of waves by a nickel cylinder. And we conclude in Section IV.

\section{METHODS}

\section{A. Theory}

The theory used for computing our simulation is based on the formalism developed by Mie for bodies of spherical shapes [3] and later developed for cylindrical geometries [4], [5]. The theory gives a rigorous solution

Submitted on November 12, 2021

Published on January 26, 2022.

A. Kazadi Mukenga Bantu, Department of Physics, University of Kinshasa, Kinshasa, Democratic Republic of Congo; Department of Physics, Catholic University of Louvain-la-Neuve, Louvain-la-Neuve, Belgium.

(e-mail: albert.kazadi@ unikin.ac.cd)

T. Mabiala Masiala, Department of Physics, University of Kinshasa, Kinshasa, Democratic Republic of Congo; Department of Physics,

Catholic University of Louvain-la-Neuve, Louvain-la-Neuve, Belgium.

E. Phuku Phuati, Department of Physics, University of Kinshasa, Kinshasa, Democratic Republic of Congo.

J. P. Mbungu Tsumbu, Department of Physics, University of Kinshasa, Kinshasa, Democratic Republic of Congo.

P. Defrance, Department of Physics, Catholic University of Louvain-la-Neuve, Louvain-la-Neuve, Belgium. 
of the Maxwell equations for a plane monochromatic wave impinging a cylinder under any incidence with respect to the cylinder axis. From this solution expressed as a series of terms the components of the internal field and that of the scattered field can be determined by the means of some proportionality coefficients called the Mie coefficients for the respective fields. From the field components, the intensity can be deduced.

In the present work, we are interested in the far-field distribution of the intensity around the cylinder. The general solution of the Maxwell scalar equation in cylindrical coordinates is given in the form:

$$
\psi_{n}(r, \theta, z)=\exp (i \omega t) Z_{n}(j r) \exp (i n \theta) \exp (-i h z)
$$

From the scalar solutions of the wave equation, the vector fields can be rebuilt through these auxiliary fields:

$$
\begin{aligned}
& \vec{M}_{\psi}=\vec{\nabla} \times\left(\vec{a}_{z} \psi\right)=\vec{a}_{r} \frac{1}{r} \frac{\partial \psi}{\partial \theta}-\vec{a}_{\theta} \frac{\partial \psi}{\partial r} \\
& \vec{N}_{\psi}=\frac{1}{m k}\left(\vec{\nabla} \times \vec{M}_{\psi}\right)=\vec{a}_{r} \frac{\partial^{2} \psi}{\partial z \partial r}+\vec{a}_{\theta} \frac{1}{r} \frac{\partial^{2} \psi}{\partial z \partial \theta}-\vec{a}_{z}\left[\frac{1}{r} \frac{\partial}{\partial r}\left(r \frac{\partial \psi}{\partial r}\right)+\frac{1}{r^{2}} \frac{\partial^{2} \psi}{\partial \theta^{2}}\right]
\end{aligned}
$$

These fields are linear combination of the electromagnetic field which is solution of the vector field wave equations:

$$
\begin{aligned}
& \vec{E}=\vec{M}_{v}+i \vec{N}_{u} \\
& \vec{H}=m\left(-\vec{M}_{u}+i \overrightarrow{N_{v}}\right)
\end{aligned}
$$

The entire field is determined through boundary conditions at the cylinder interface with the outer medium. This is stated by the fact the tangential and normal components of the fields must be continuous at this interface. It is expressed as:

$$
\begin{aligned}
& E_{\theta}^{i}+E_{\theta}^{s}=E_{\theta}^{t} ; H_{\theta}^{i}+H_{\theta}^{s}=H_{\theta}^{t} \\
& E_{z}^{i}+E_{z}^{s}=E_{z}^{t} ; H_{z}^{i}+H_{z}^{s}=H_{z}^{t}
\end{aligned}
$$

\section{Here $r=a$.}

In this way, the scattered field and the internal field are proportional to the incident field through coefficients of proportionality. These coefficients are called the Mie coefficients for the scattered field and the internal field, respectively. These are found by solving the linear system incurred by the application of the boundary conditions. In that manner the scattered field can be expressed in the far field region:

$$
\begin{aligned}
& u^{s} \cong \sqrt{2 / \pi l r} \exp [i(\omega t-h z-l r)-i 3 \pi / 4] \sum_{n} b_{n 1} \cos (n \theta) \\
& v^{s} \cong \sqrt{2 / \pi l r} \exp [i(\omega t-h z-l r)-i 3 \pi / 4] \sum_{n} a_{n 1} \sin (n \theta)
\end{aligned}
$$

The scalar functions, solution of the wave equation are given by:

$$
\begin{aligned}
& u^{s} \cong \sqrt{2 / \pi l r} \exp [i(\omega t-h z-l r)-i 3 \pi / 4] \sum_{n} b_{n 1} \cos (n \theta) \\
& v^{s} \cong \sqrt{2 / \pi l r} \exp [i(\omega t-h z-l r)-i 3 \pi / 4] \sum_{n} a_{n 1} \sin (n \theta)
\end{aligned}
$$

Neglecting terms of degrees above $(1 / r)^{1 / 2}$, the components may be written as:

$$
\begin{aligned}
& E_{r}^{s} \cong-(i k \sin \alpha \cos \alpha) u^{s} \\
& E_{\theta}^{s} \cong(i k \cos \alpha) v^{s} \\
& E_{z}^{s} \cong(i k \cos \alpha) u^{s}
\end{aligned}
$$

The expression of the far-field scattered intensity for an infinite cylinder under normal incidence is finally given in the form:

For case I ( $\vec{E}$ parallel to the cylinder axis): 
$l_{11}=\frac{l_{0}}{\pi k R}\left|b_{01}+2 \sum_{n} b_{n 1} \cos (n \theta)\right|^{2}$

For the case II ( $\vec{E}$ perpendicular to the cylinder axis):

$$
l_{22}=\frac{l_{0}}{\pi k R}\left|a_{02}+2 \sum_{n} a_{n 2} \cos (n \theta)\right|^{2}
$$

Where $a_{n 2}$ and $b_{n 1}$ are the Mie coefficients corresponding to the $\mathrm{n}^{\text {th }}$ order TM and TE mode respectively for the scattered field.

They are given as follow:

$$
\begin{aligned}
& a_{n 2}=\frac{m J_{n}(y) J_{n}^{\prime}(x)-J_{n}(x) J_{n}^{\prime}(y)}{m J_{n}(y) H_{n}^{2^{\prime}}(x)-H_{n}^{2}(x) J_{n}^{\prime}(y)} \\
& b_{n 1}=\frac{J_{n}(y) J_{n}^{\prime}(x)-m J_{n}(x) J_{n}^{\prime}(y)}{J_{n}(y) H_{n}^{1^{\prime}}(x)-H_{n}^{1}(x) J_{n}^{\prime}(y)}
\end{aligned}
$$

The lower indices (1) and (2) in the coefficients refer to the case I (E parallel to the cylinder axis) and to case II (E orthogonal to cylinder axis). And $(\mathrm{m})$ is the relative refractive index of the scattering material, $\mathrm{J}_{\mathrm{n}}(\mathrm{y})$ is the cylindrical $n^{\text {th }}$ order Bessel function for $n=0,1,2$, the Hankel function of the first kind, $\mathrm{H}_{\mathrm{n}}{ }^{(1)}$ or the Hankel function of the second kind, $\mathrm{H}_{\mathrm{n}}{ }^{(2)}$ respectively. The $\mathrm{x}=2 \pi \mathrm{R} / \lambda$ (with $\mathrm{R}=$ radius of nickel metal cylinder) is the size parameter and $(\mathrm{y}=\mathrm{mx})$ is the product of the refractive index with the size parameter.

Knowing these elements, the far-field intensity distribution can be computed using a code simulation.

\section{B. Simulation}

In the present study, we have computed a code rendering the distribution for an infinite cylinder under normal incidence. We have developed a MATLAB code that can be run on a personal computer.

The input parameters are the cylinder diameter, the incident wavelength and the complex refractive Index of the material at the given wavelength. The starting point is the Mie coefficients given in the preceding sections.

The code that gives these coefficients is:

$\mathrm{x}=1000 ; \mathrm{m}=$ complex $(1.54000,0.00) ; \mathrm{k}=1800 ; \% \mathrm{x}$ : size parameter, $\mathrm{m}$ : complex refractive index

$\operatorname{nmax}=\operatorname{round}\left(2+\mathrm{x}+4 * \mathrm{x}^{\wedge}(1 / 3)\right)$;

$\mathrm{n}=(1: \mathrm{nmax}) ; \mathrm{nu}=(\mathrm{n}) ; \mathrm{z}=\mathrm{m} . * \mathrm{x}$;

$\mathrm{a} 01=(\operatorname{besselj}(-1, \mathrm{z}) * \operatorname{besselj}(0, \mathrm{x})-\mathrm{m} * \operatorname{besselj}(0, \mathrm{z}) * \operatorname{besselj}(-1, \mathrm{x}))$;

a02 $=(\operatorname{besselj}(-1, \mathrm{z}) * \operatorname{besselh}(0, \mathrm{x})-\mathrm{m} * \operatorname{besselj}(0, \mathrm{z}) * \operatorname{besselh}(-1, \mathrm{x}))$;

$\mathrm{a} 0=\mathrm{a} 01 . / \mathrm{a} 02$;

b01 $=\left(\mathrm{m}^{*} \operatorname{besselj}(-1, \mathrm{z}) * \operatorname{besselj}(0, \mathrm{x})\right.$-besselj $\left.(0, \mathrm{z}) * \operatorname{besselj}(-1, \mathrm{x})\right)$;

b02 $=(\mathrm{m} * \operatorname{besselj}(-1, \mathrm{z}) * \operatorname{besselh}(0, \mathrm{x})-\operatorname{besselj}(0, \mathrm{z}) * \operatorname{besselh}(-1, \mathrm{x}))$;

b0=b01./b02;

$\operatorname{sqx}=\operatorname{sqrt}(0.5 *$ pi. $/ \mathrm{x}) ; \operatorname{sqz}=\operatorname{sqrt}(0.5 *$ pi. $/ \mathrm{z})$;

$\mathrm{bx}=\operatorname{besselj}(\mathrm{nu}, \mathrm{x})$;

$\mathrm{bz}=\operatorname{besselj}(\mathrm{nu}, \mathrm{z})$;

$\mathrm{yx}=\operatorname{bessely}(\mathrm{nu}, \mathrm{x})$;

$\mathrm{hx}=$ complex $(\mathrm{bx}, \mathrm{yx})$;

$\mathrm{b} 1 \mathrm{x}=[\sin (\mathrm{x}) / \mathrm{x}, \mathrm{bx}(1: \mathrm{nmax}-1)]$;

$\mathrm{b} 1 \mathrm{z}=[\sin (\mathrm{z}) / \mathrm{z}, \mathrm{bz}(1: \mathrm{nmax}-1)]$;

$\mathrm{y} 1 \mathrm{x}=[-\cos (\mathrm{x}) / \mathrm{x}, \mathrm{yx}(1: \operatorname{nmax}-1)]$;

$\mathrm{h} 1 \mathrm{x}=$ complex $(\mathrm{b} 1 \mathrm{x}, \mathrm{y} 1 \mathrm{x})$;

$\mathrm{ax}=(\mathrm{x} . * \mathrm{~b} 1 \mathrm{x}-\mathrm{n} . * \mathrm{bx}) . /(\mathrm{x})$;

$\mathrm{az}=(\mathrm{z} \cdot * \mathrm{~b} 1 \mathrm{z}-\mathrm{n} . * \mathrm{bz}) \cdot /(\mathrm{z})$;

$\mathrm{ahx}=\left(\mathrm{x} . *^{*} \mathrm{~h} 1 \mathrm{x}-\mathrm{n} \cdot{ }^{*} \mathrm{hx}\right) \cdot /(\mathrm{x})$;

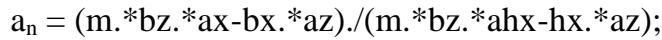

$\mathrm{b}_{\mathrm{n}}=(\mathrm{bz} \cdot * \mathrm{ax}-\mathrm{m} . * \mathrm{bx} . * \mathrm{az}) . /(\mathrm{bz} \cdot * \mathrm{ahx}-\mathrm{m} . * \mathrm{hx} . * \mathrm{az})$

Here $a_{n}$ and $b_{n}$ are the Mie coefficients [6] corresponding to the $\mathrm{n}^{\text {th }}$ order TM and TE mode respectively, for the cylinder scattered field. Although the sum of the series is infinite, in the computation we use a maximum value from which the series converges: this value has been calculated by Wiscombe [7]. 
The second step is the expression of the partial wave series, in which the absolute square of the sum gives the far-field distribution of the intensity around the cylinder.

The module below gives the lines of the code:

$\mathrm{i}=1: \mathrm{nmax} ; \mathrm{j}=1: \mathrm{k} ; \mathrm{dtheta}=180 . /(\mathrm{k}-1) ;$ theta $=(\mathrm{j}-1) *$ dtheta;

for $n=1:(n \max -1) ; Z(n, j)=2 * \operatorname{cosd}(n *($ theta $))$; end

$\mathrm{l}=1: \mathrm{k} ; \mathrm{I}(1, \mathrm{l})=2$;

$\mathrm{Z} 1=[\mathrm{I} ; \mathrm{Z}]$;

$\mathrm{A}=\mathrm{an} ; \mathrm{B}=\mathrm{bn}$;

for $\mathrm{i}=1: n \max ; A Z 1(i, j)=(A(1, i)) *(Z 1(i, j)) ;$ end

for $\mathrm{i}=1: \mathrm{nmax} ; \mathrm{BZ1}(\mathrm{i}, \mathrm{j})=(\mathrm{B}(1, \mathrm{i})) . *(\mathrm{Z} 1(\mathrm{i}, \mathrm{j})) ;$ end

$\mathrm{S} 1=\mathrm{a} 0+\operatorname{sum}(\mathrm{AZ} 1) ; \mathrm{S} 2=\mathrm{b} 0+\operatorname{sum}(\mathrm{BZ} 1)$;

$\mathrm{YS} 1=\mathrm{abs}(\mathrm{S} 1) ; \% \mathrm{YS} 1$ corresponds to case II(E orthogonal to cylinder axis )

$\mathrm{YS} 2=\mathrm{abs}(\mathrm{S} 2)$; \% YS2 corresponds to case I(E parallel to cylinder axis)

plot(theta, YS1,theta, YS2); grid on;

Our computations have been limited to a size parameter $\mathrm{x}$ of 179.00 which corresponds to a diameter of $36.05 \mu \mathrm{m}$ for an incident wavelength of $632.8 \mathrm{~nm}$ (typical He-Ne laser source wavelength).

\section{Experiment}

The experiment has been carried out using a linearly polarized He-Ne Laser emitting at $632.8 \mathrm{~nm}$ as the incident source, a metalized nickel cylinder of $335 \mu \mathrm{m}$ as the target wire, the intensity distribution has been recorded using a CCD camera Thorlabs ${ }^{\circledR}$, model DUC 223 as shown in Fig. 1.
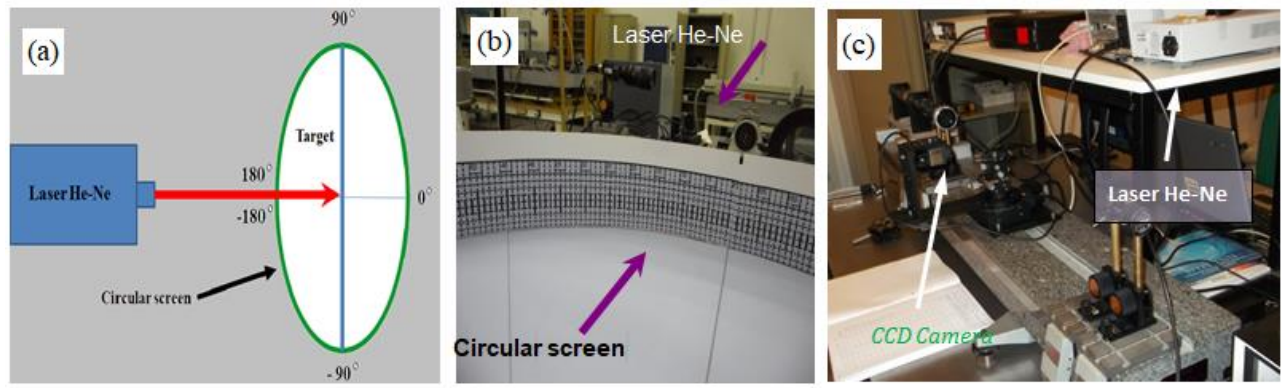

Fig. 1 (a) schematic representation of the first experimental set-up, (b) view of circular screen and Laser source and (c) global view of second experimental set-up using CCD camera for intensity recording.

The micrograph of the sample has been taken using a Zeiss ${ }^{\circledR}$ optical microscope, and the radius of the cylinder was measured using the rule tool of the microscope capturing software. The angular position has been recorded using a Thorlabs ${ }^{\circledR}$ nano-rotator. The scattering geometry and Micro-photographs of samples used are shown in Fig. 2. We consider a nickel metal cylindrical scatterer, with the cylinder axis aligned with the anisotropy axis (taken to be the $z$-axis). In the Fig. 2 a, we show the incidence angle $\theta$, which is defined as the angle between the incident wave vector $k_{\text {inc }}$ and the $z$-axis. We assume that the incident wave vector has a $z$ component $k_{z}$. The dielectric cylinder has radius $R$.
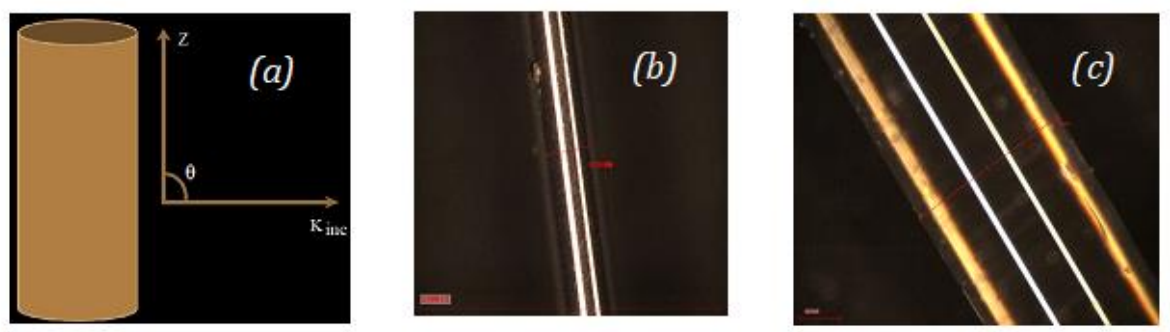

Fig. 2. (a) Scattering of plane wave by a dielectric Nickel metalized cylinder aligned with the anisotropy axis along the $\mathrm{z}$-axis. Microphotographs of samples used in our experiment with total diameter (b) of $0.35 \mathrm{~mm}$ for Nickel metalized cylinder of $1.00 \mu \mathrm{m}$ thickness and (c) of $2.03 \mathrm{~mm}$ for low-lost dielectric fused silica cylinder.

\section{RESUlTS AND DisCUSSION}

Fig. 3a shows the intensity distribution around the Nickel metalized cylinder. Experiment data are in grey color, Lorenz-Mie model in light blue, and Fresnel model in green color. The incident electric field is parallel to the cylinder axis. And in Fig. $3 \mathrm{~b}$ the incident electric field is perpendicular to the cylinder axis. 


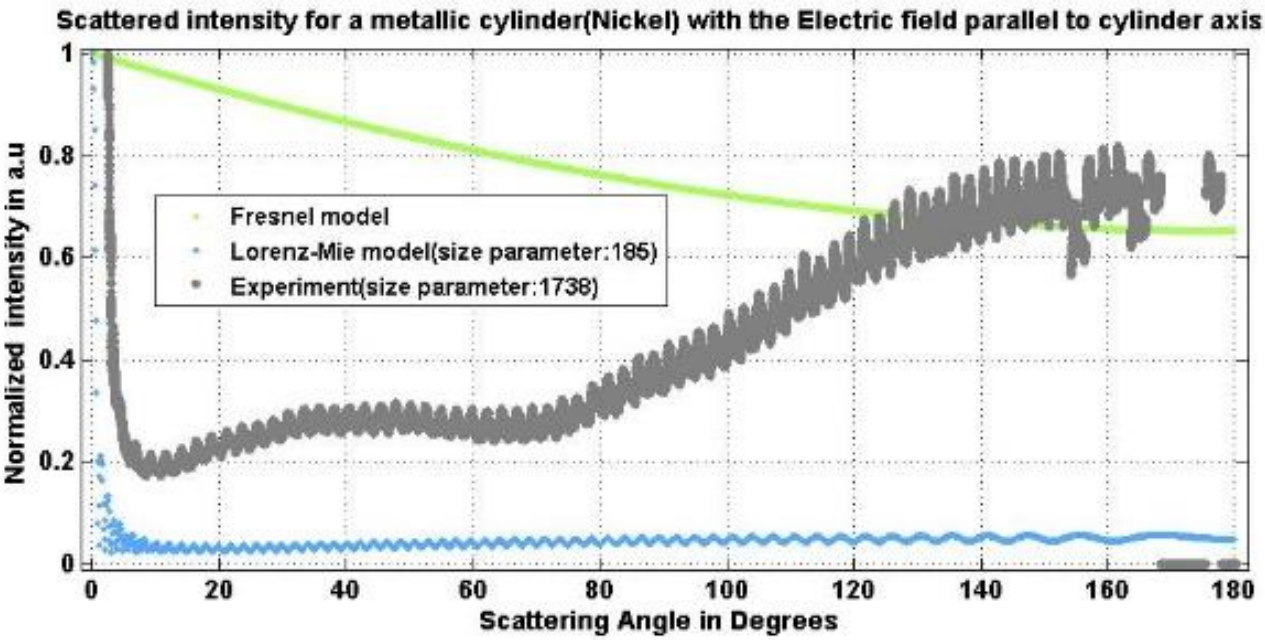

Fig. 3a. Intensity distribution around a Nickel cylinder. Experiment (grey), Lorenz-Mie model (light blue), Fresnel model (green). The incident electric field is parallel to cylinder axis

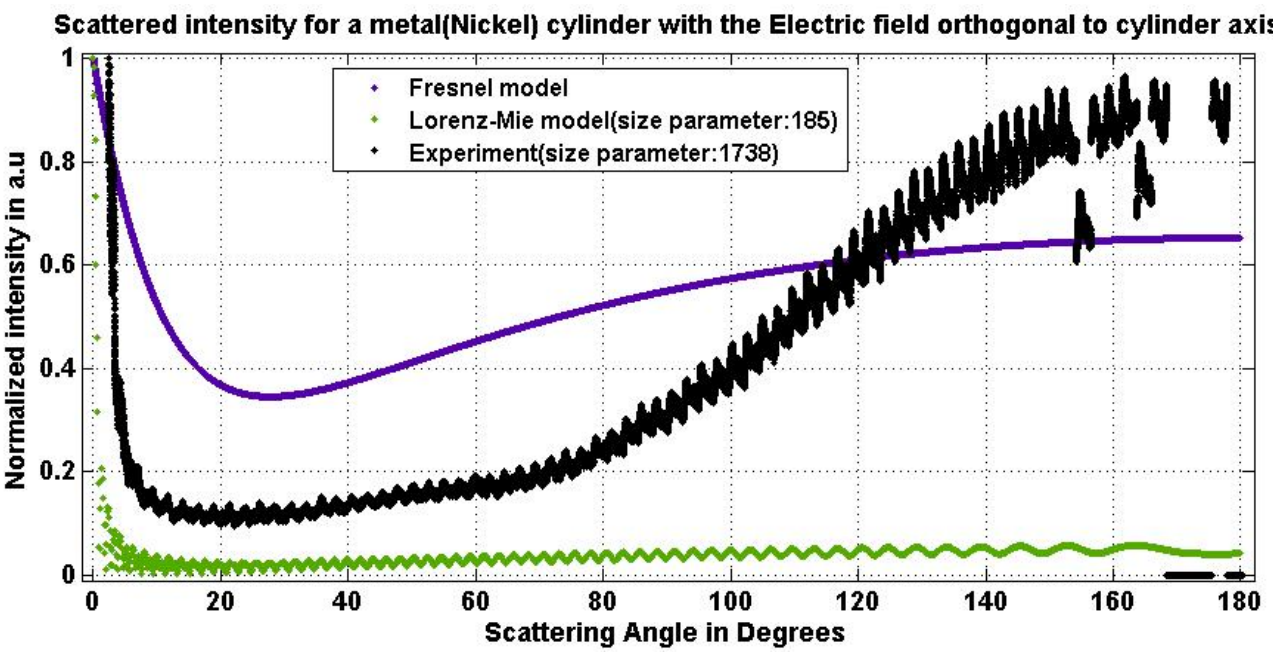

Fig. 3b. Intensity distribution around a Nickel cylinder. Experiment (black), Lorenz-Mie model (green), Fresnel model (dark blue). The incident electric field is orthogonal to cylinder axis.

In Fig. 4 we compare the experimental data with the Fresnel model (Fresnel Reflectance Spectra). This Fig. 4 shows the angular distribution of the scattered intensity.

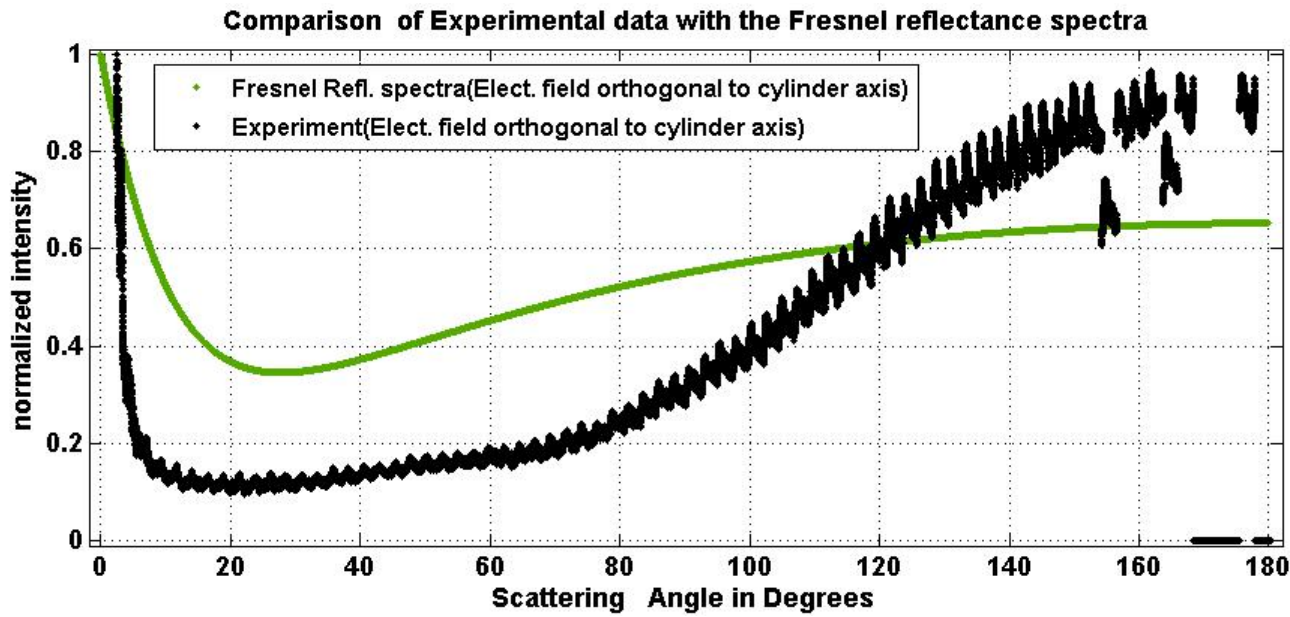

Fig. 4a. Angular distribution of the scattered intensity. Fresnel model (green), experiment (black). Electric field orthogonal to cylinder axis.

One could observe that in Fig. 4a the Fresnel model is in green color and the experimental data in black. Here the electric field is orthogonal to the cylinder axis. 


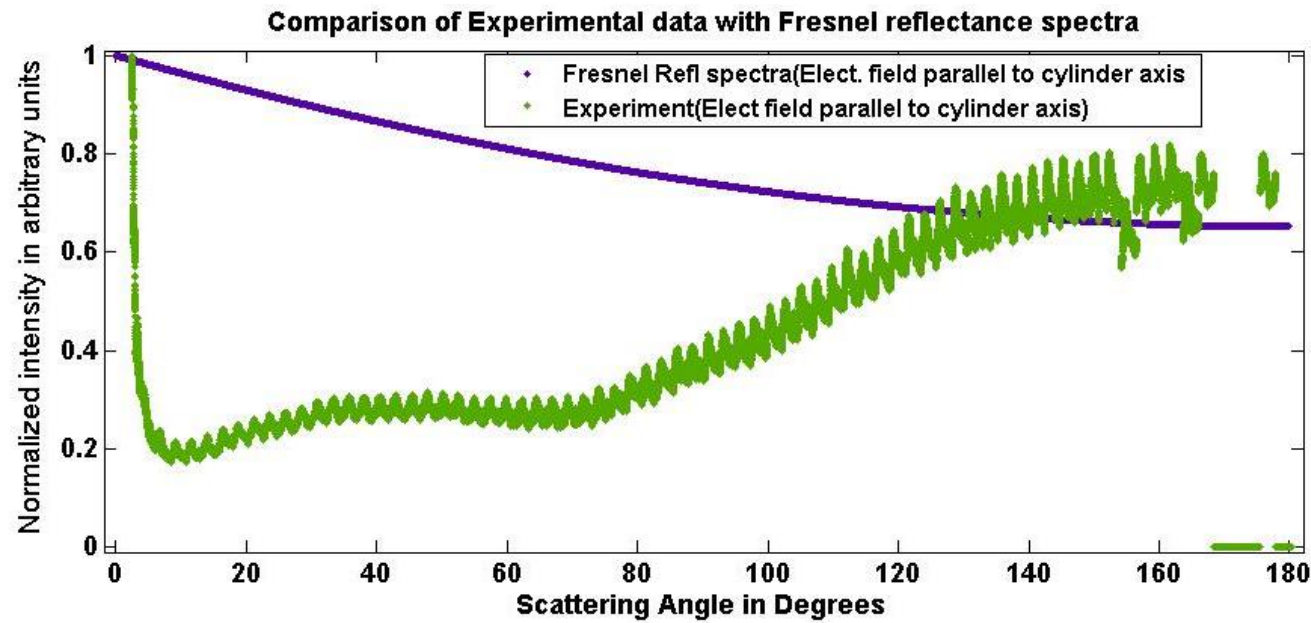

Fig 4b. Angular distribution of the scattered intensity. Fresnel model (dark blue), experiment (green). Electric field parallel to cylinder axis.

And in Fig. 4b the Fresnel model is in dark blue color and the experiment is in green. The electric is field parallel to the cylinder axis. From Fig. 3a, 4a and 3b, 4b, the far-field intensity accuses a rapid decrease in the intensity profile in the vicinity of the forward direction, diffraction is quite noticeable in experiments, but with a slight difference in the maxima separation for the same size parameter, narrower than for the absorbing cylinder. This means a systematic overestimation of the diameter using Fraunhoffer approximation. The experiment shows a quite noticeable intensity distribution in the whole scattering range with a strong component in the backward region, this is due certainly to the strong reflectivity of metals in the optical range. A minimum appears in the intensity distribution at an angle corresponding to that of Brewster for the material, a comparison is made with the Fresnel model for both polarizations. The Lorentz-Mie model shows that the Fresnel model is a good approximation for large cylinders, especially for studying the distribution of linear polarization. As the size parameter decreases the scattering regime also changes tending to become, as in the preceding case uniform. But the fading of one the polarization is more important than that of the absorbing cylinder. The distribution becomes uniform for size parameters equals or lowers than one, reaching value of $70 \%$. Nano-sized metallic cylinders are good polarizer.

Fig. 5 shows the distribution of linear polarization for the Lorenz-model, Fresnel model and experiment data.

Fig. 5a shows that the Fresnel model renders well the distribution of linear polarization for large metallic cylinders, except in the forward direction where there are strong oscillations in that distribution. This polarization is due to the shift of maxima and minima for each polarization. This behavior has been studied theoretically by Sanchez et al.[8].

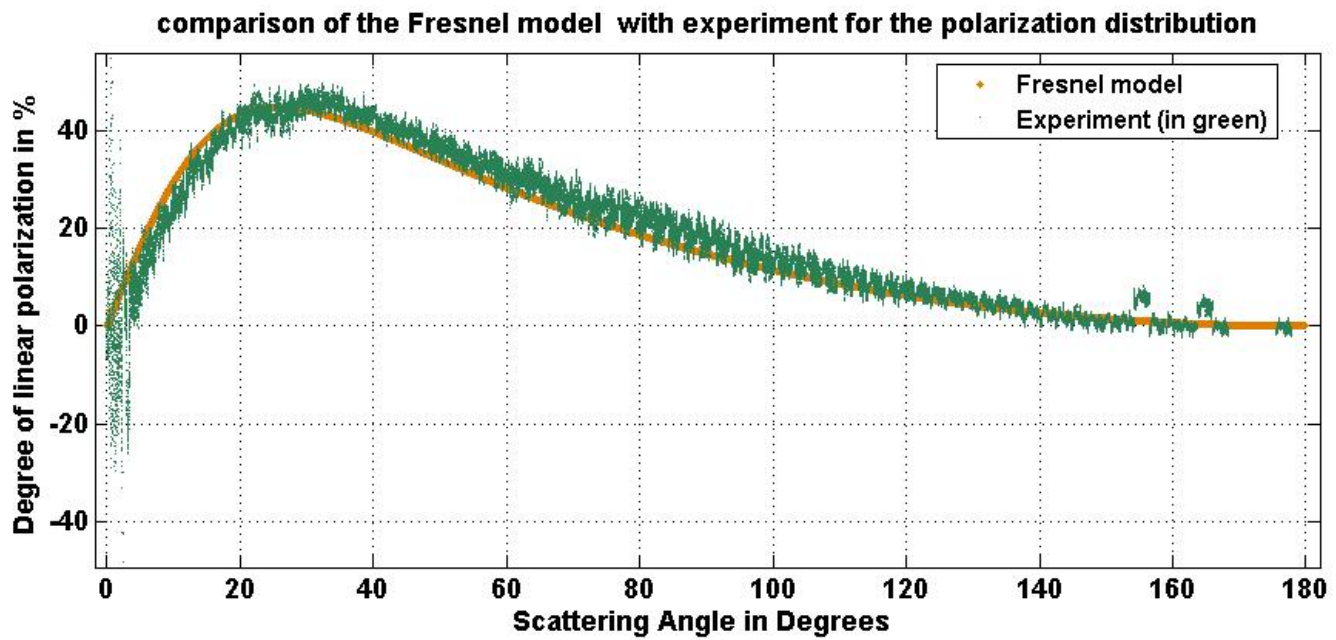

Fig. 5a. Distribution of linear polarization for the Fresnel model (brown), and experiment (green)

The Lorenz Mie model shows (Fig. 5b) that, due to the change of scattering regime with the parameter size, the polarization distribution also changes. This changes the Fresnel model which is unable to follow the experimental data. The model shows also that there are oscillations in the polarization distribution due mostly to the dependence of maxima and minima position with respect to the polarization state of light. 


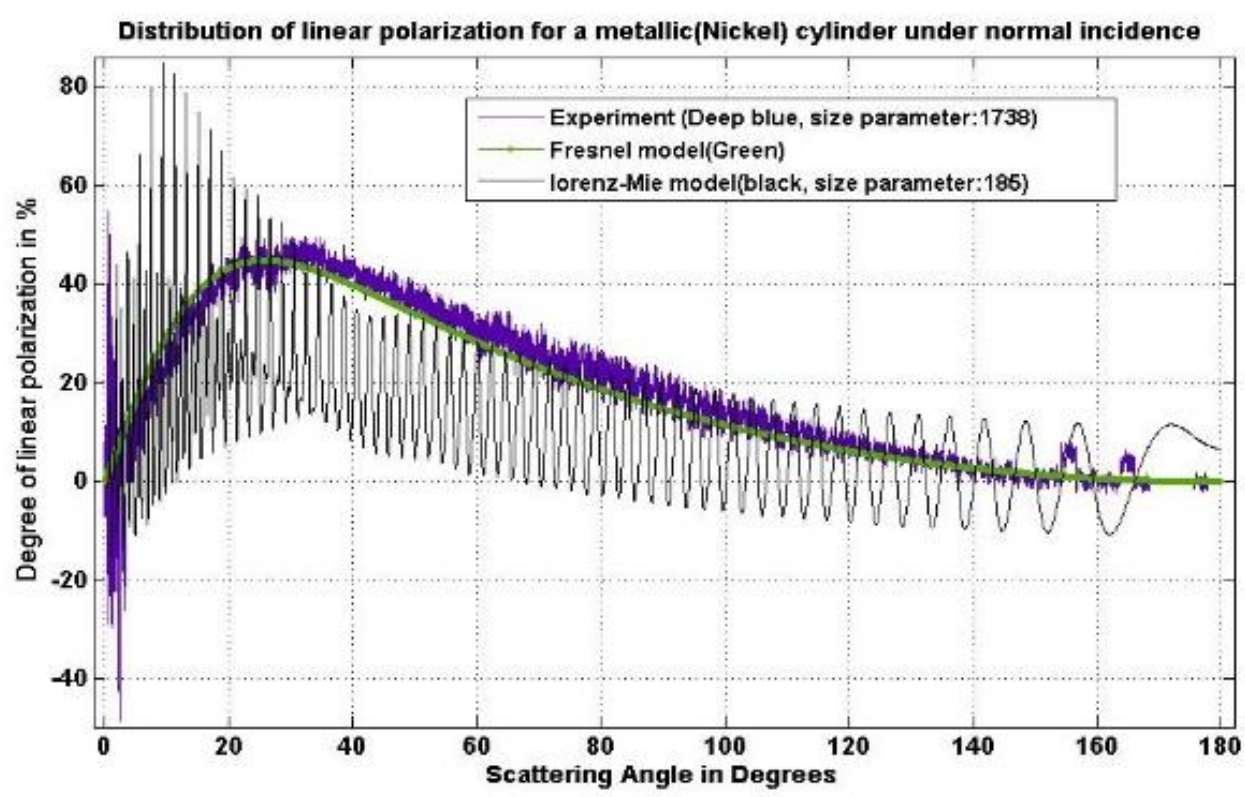

Fig. 5b. Distribution of linear polarization for the Lorenz-model (black), Fresnel model (green), and experiment (dark blue).

Fig. 6 shows the interference fringes images for each polarization in the forward direction, in the geometrical section. Left (Electric field parallel to nickel cylinder axis), right (Electric field orthogonal to nickel cylinder axis). One could observe on it, in the geometrical shadow, a series of interference fringes in place of intensity pic as in the case of an opaque target (diffraction) or in the darkness region. Table I shows the angular separation between successive maxima (or minima). These interferences lines could be explained by taking the quartz cylinder as a circular lens. The interferences pattern could be due to the walking difference between different light rays that have undergone different orders of reflection before emerging in the common direction. Fig. 7 shows the scattered pattern in the forward direction. Note the phase opposition between the polarization. The exam of these interferences shows that the position of minima and maxima is inverted when passing from one polarization to another.
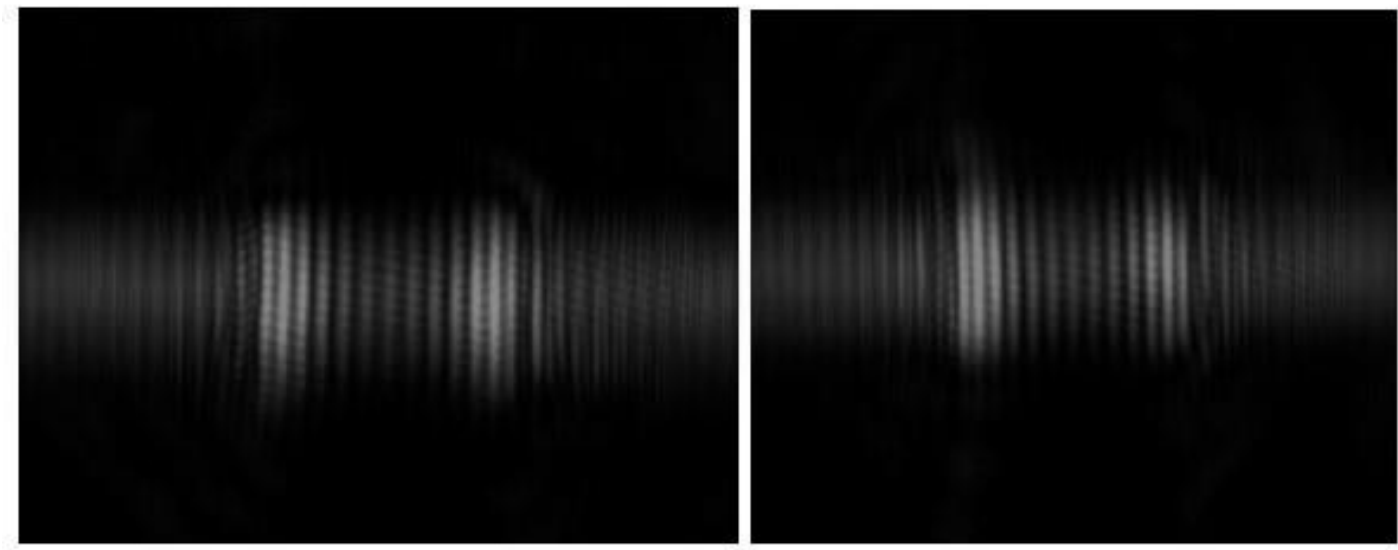

Fig. 6. Interference pattern in the forward direction, in the geometrical section. Left (Electric field parallel to nickel cylinder axis), right (Electric field orthogonal to nickel cylinder axis).

TABLE I: ANGUlar SEPARATION BETWEen SUCCESSIVE MAXIMA (OR Minima) IN THE INCIDENT WAVE DiRECTION

\begin{tabular}{cccccccccc}
\hline \multicolumn{7}{c}{ Gap between successive maxima (parallel polarization) } \\
\hline 5 & 4 & 3 & 2 & 1 & 2 & 3 & 5 \\
0.0626 & 0.0719 & 0.07409 & 0.07413 & 0.07413 & 0.07415 & 0.0718 \\
\hline \multicolumn{7}{c}{ Gap between successive minima (orthogonal polarization) } \\
\hline 5 & 4 & 3 & 2 & 1 & 2 & 3 & 0.0625 \\
0.0672 & 0.0718 & 0.07414 & 0.08108 & & 0.07877 & 0.06915 & 0.0672 & 0.0625 \\
\hline
\end{tabular}




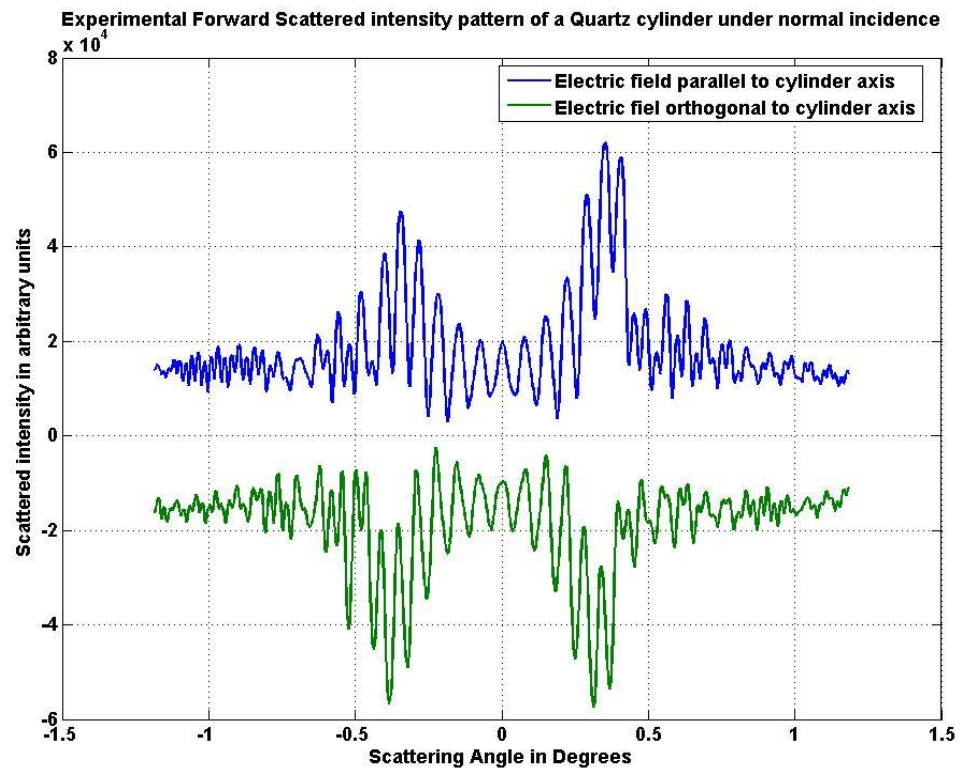

Fig. 7. Scattered pattern in the forward direction. Note the phase opposition between the minima and maxima when they pass from one polarization to another.

\section{CONCLUSION}

We have investigated the scattering of an incident monochromatic plane wave, on a nickel metallic cylinder. The investigation was carried out through two simulation codes written to simulate the intensity distribution. One of the codes was based in the Lorenz-Mie solution for a cylindrical shape; another was based on the Fresnel reflection coefficients adapted for cylindrical shapes bodies. The investigation was completed by experiments involving a Nickel cylinder impinged by a linearly polarized He-Ne laser beam. The results show that the Fresnel model is unsuited to describe intensity distribution for small sized cylinders. For large cylinder, however, the model is a little closer to the intensity distribution displayed by the metallic cylinder. The linear polarization distribution is well described by this model, except in the angular range of $0^{\circ}$ to $5^{\circ}$ where the experiment displays strong oscillation of the linear polarization. The Lorenz-Mie model also displays the same behavior. In brief, the Fresnel model is only suited to describe linear polarization for large cylinders. For medium sized and small cylinders, it is better to use the Lorenz-Mie model because it can follow the evolution of the scattering regime through different size parameter ranges. Experimental studies would be now under way to confirm these modeled results in the future.

\section{ACKNOWLEDGENT}

The authors acknowledge financial support from the University Commission for Development (CUD) in partnership with the Belgium French-speaking Universities (Grant No. Kin 04).

\section{REFERENCES}

[1] Rituraj Peter B. Catrysse and Shanhui Fan. Scattering of electromagnetic waves by cylinder inside uniaxial hyperbolic medium, Opt. Express 2019;27(4):3991-4003.

[2] Jackson J. D., Electrodynamique classique,. s.1.: édition DUNOD, 1995.

[3] G. Mie., Beiträge zur Optik trüber Medien, speziell kolloidaler Metallösungen. Ann Physik, 1908; 25:377-455.

[4] Van de Hulst H.C. Light scattering by small particles, edited by John Wiley \& sons, Inc., New york,USA, 1957, p. 395. chapter 9.

[5] Kuo Nan Liou. Electromagnetic scattering by arbitrarily oriented ice cylinders, [éd.] Optical society of America., J. Applied Optics, March 1972;11:667.

[6] C. F. Bohren and D. R. Huffman. Absorption and Scattering of Light by Small Particles, Wiley-VCH, 1998.

[7] Wiscombe W.J. Improved Mie Scattering Algorithms. Applied Optics, 1980;19:1505-1509.

[8] L. Miguel Sanchez Bréa, J.C. Martinez, E. Barnabeu. Effect of the refraction index in the diameter estimation of thin metallic wires. [éd.] SPIE. Bellingham,WA: s.n., Proceedings of SPIE, vol. 5858, 2005. 